\title{
Comparison Analysis of Single Loop Resonator Based Miniaturized Triple-Band Planar Monopole Antennas
}

\author{
Kaimin Wu, Yongjun Huang, Ruiming Wen, Jian Li, and Guangjun Wen \\ Centre for RFIC and System Technology, School of Communication and Information Engineering, \\ University of Electronic Science and Technology of China, Chengdu 611731, China \\ Correspondence should be addressed to Yongjun Huang; yongjunh@uestc.edu.cn
}

Received 16 September 2014; Revised 21 December 2014; Accepted 24 December 2014

Academic Editor: Sanming Hu

Copyright (c) 2015 Kaimin Wu et al. This is an open access article distributed under the Creative Commons Attribution License, which permits unrestricted use, distribution, and reproduction in any medium, provided the original work is properly cited.

\begin{abstract}
We systemically analyze and discuss a group of miniaturized triple-band planar monopole antennas by integrating with a single loop resonator (SLR) as part of the radiation patch. Two configurations (rectangular and circular shaped radiation patches) are presented and the corresponding SLRs are integrated in the patches to contribute additional operating frequencies. Two feed techniques (coplanar waveguide and microstrip feeds) are performed to couple the electromagnetic waves. The conventional planar monopole antennas without the SLRs are also analyzed comparatively. Numerical and experimental methods are performed to demonstrate and discuss the proposed designs.
\end{abstract}

\section{Introduction}

In recent years, due to the breakthroughs of modern wireless communications, for example, near field communications (NFC), radio frequency identifications (RFID), and the internet of things (IoT), the developments of miniaturized and multiband/multimode communication components have been the main barriers in such science and engineering areas. The first component, for instance, used to send/receive the electromagnetic wave signals should be the antenna component, and comprehensive researches have focused on the multiband antennas and/or ultrawideband antennas, for the miniaturization and multiband/multimode requirements. In the various kinds of reported multiband antennas, monopole antenna is one of the most attractive configurations in recent years, because of the compact sizes, flexible designs, low fabrication costs, and well radiation characteristics. Some of the monopole antenna configurations are, for example, circular/rectangular/spiral ring patch antenna [1-3], I-shaped/Ushaped slot defected [4] and T-shaped slot defected planar antenna [5], and spirograph planar antenna [6]. These multiband monopole antennas were achieved by properly designing various resonator configurations to reduce operating frequencies, improve radiation pattern, and at the same time reduce cross-polarization characteristics. However, most of these antennas have complex configurations.

On the other hand, since the realization of the first metamaterial in 2000 [7], it has been proposed that the bulk metamaterials or metamaterial units can be widely used for designing novel antennas to improve most of the characteristics comparing with conventional antennas [8]. For the monopole antenna integrated with metamaterial unit, some novel configurations have been reported elsewhere, for example, the band-notched ultra-wideband (UWB) planar antennas with a modified complementary split-ring resonator [9] and the single metamaterial unit inspired dualband/multiband monopole antennas [10-12]. Taking into deep consideration the previously mentioned metamaterialinspired dual/multiband antennas, two or three different resonators were integrated as the radiation part to achieve dual/multiband properties. In this paper, we propose and systematically analyze a group of miniaturized triple-band planar monopole antennas by integrating with a single loop resonator (SLR) as part of the radiation patch. Specifically, two configurations with rectangular and circular shaped radiation patches are presented and the corresponding SLRs are integrated in the patches to contribute additional operation frequencies. Two feed techniques (coplanar waveguide and 


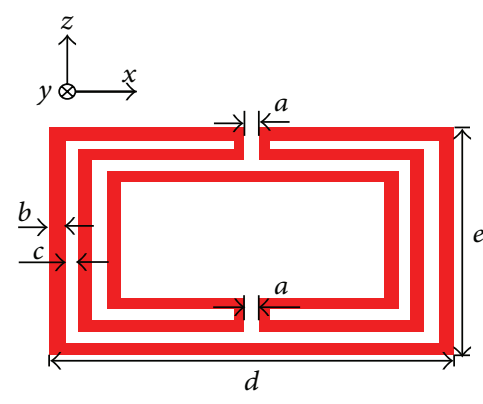

(a)

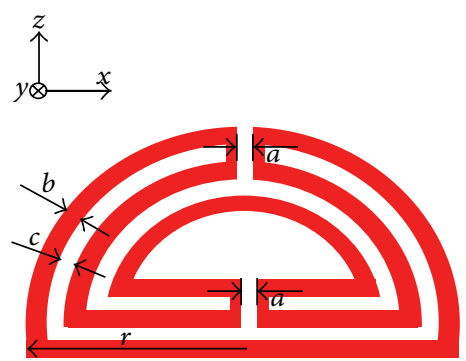

(d)

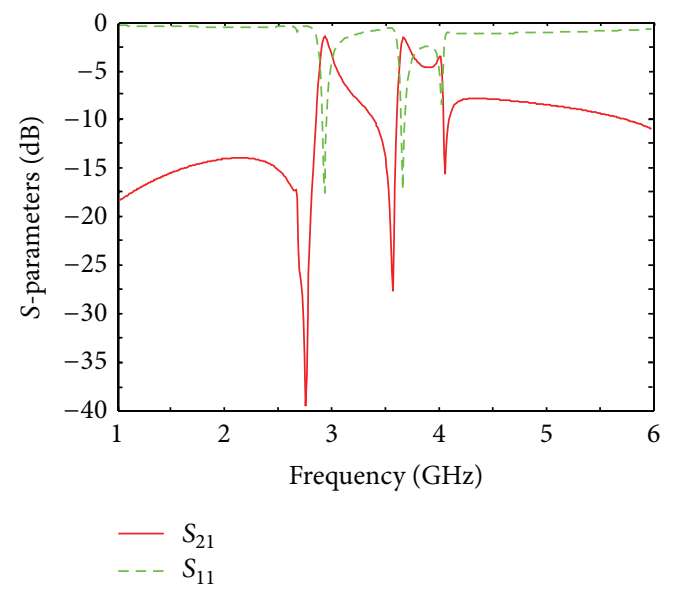

(b)

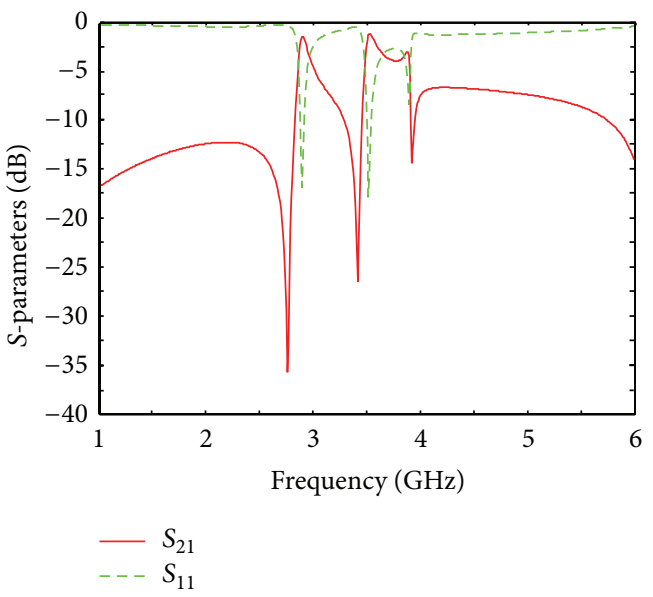

(e)

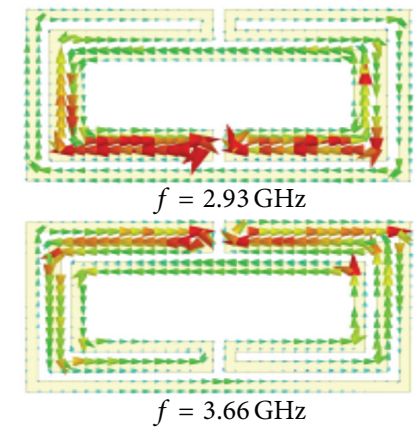

(c)

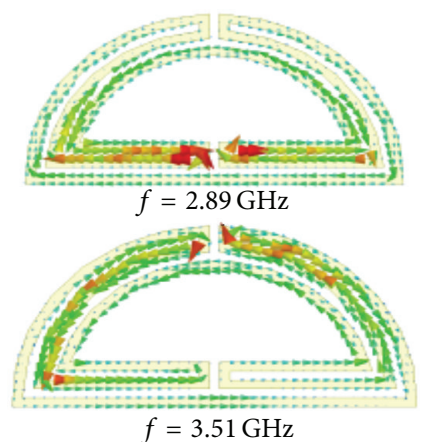

(f)

FIGURE 1: (a), (d) Unit cells of the rectangular and circular SLRs, with dimensional parameters in millimeter: $a=0.4, b=0.4, c=0.4$, $d=13.6$, and $e=6.8, r=7.8$. The period spaces along $y$-axis and $z$-axis for both cases are $12 \mathrm{~mm}$. (b), (e) Simulated transmission and reflection properties for proposed rectangular and circular SLR unit cells. (c), (f) Surface current distributions at the first two transmission dips of rectangular and circular SLRs.

microstrip feeds) are performed to couple the electromagnetic waves for the rectangular and circular shaped antennas. The conventional planar monopole antennas without the SLRs are also analyzed comparatively to show the advantageous of our proposed SLR inspired triple-band antennas. The SLR used in this paper has three or even four distinct resonances states [13-15], which therefore can be easily used to achieve the three operating frequency bands in the ranging of 2.4 to $6 \mathrm{GHz}$ [16], by properly choosing the size. We perform both numerical and experimental methods to discuss such antennas. The proposed triple-band antennas possess compact size and exhibit very well eight-like radiation patterns and low cross-polarizations, which have potential applications for modern wireless communications.

\section{Design and Fabrications}

We first briefly review the electromagnetic characteristics for the rectangular and circular SLRs as shown in Figures 1(a) and 1(d). Both cases are consisting of a single closed loop with three order meandering parts. When the electromagnetic waves act on such inclusions along the $x$-axis with the electric field along the $z$-axis, they will show two typical magnetic resonance states and a slight electric resonance state, as shown in Figures 1(b) and 1(e), respectively [13, 17]. The surface current distributions at such two magnetic resonance frequencies are shown in Figures 1(c) and 1(f) to indicate resonance characteristics. As analyzed previously [13-15], the two magnetic resonance frequencies can be flexibly adjusted by properly designing the closed loop configurations and the loop size. Therefore, one can easily integrate these SLRs into a conventional antenna. As an example, in this paper, we discuss the planar printed monopole antenna integrated with such rectangular and circular SLR units.

Based on the above introduction, the schematic geometries of the four proposed metamaterial inspired triple-band antennas and the corresponding conventional single-band monopole antennas are shown comparatively in Figure 2, 


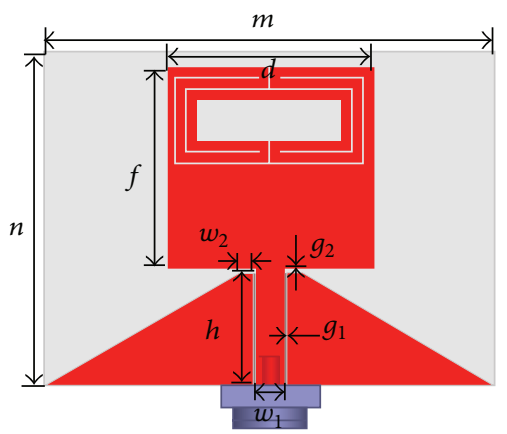

(a)

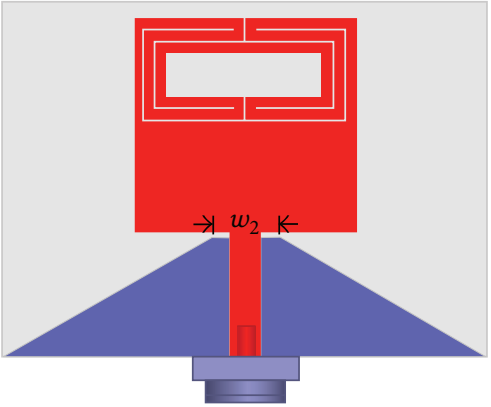

(c)

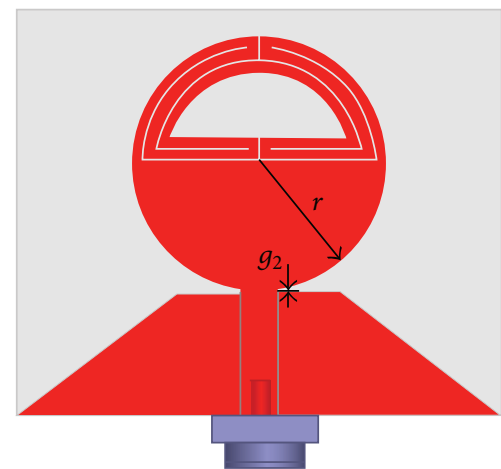

(e)

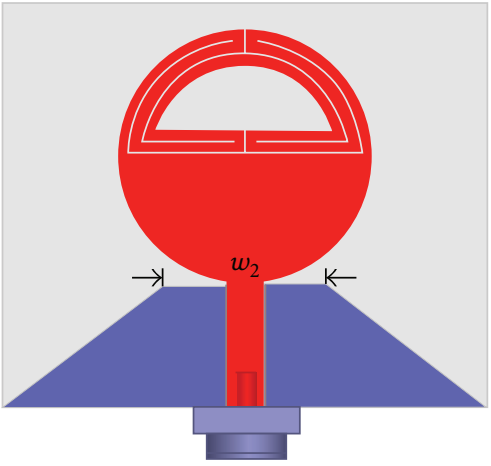

(g)

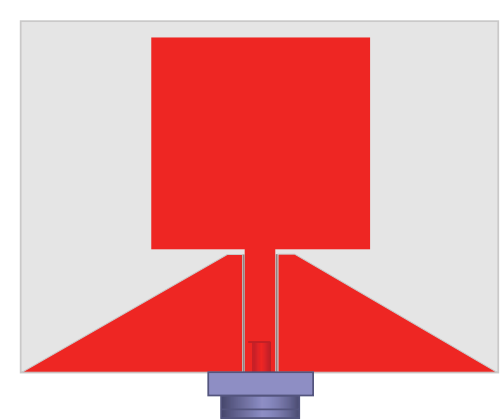

(b)

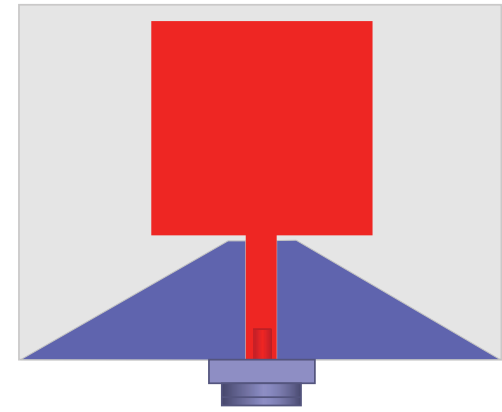

(d)

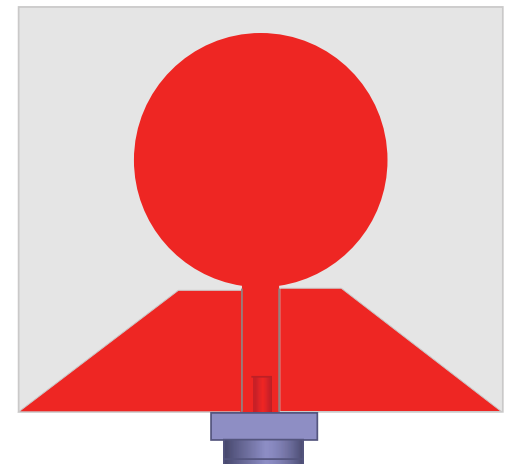

(f)

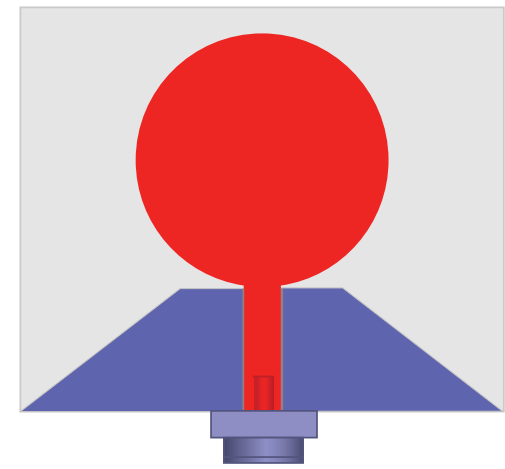

(h)

FIgURE 2: The proposed SLR inspired planar monopole antennas and corresponding conventional single band antennas. (a)-(b) CWP fed rectangular patch antennas, (c)-(d) microstrip fed rectangular patch antennas, (e)-(f) CWP fed circular patch antennas, and (g)-(h) microstrip fed circular patch antennas. 
TABLE 1: Dimensional parameters for the proposed antennas shown in Figure 2.

\begin{tabular}{|c|c|c|c|c|c|c|c|c|c|c|c|c|c|c|}
\hline Antenna & $a$ & $b$ & $c$ & $d$ & $e$ & $f$ & $g_{1}$ & $g_{2}$ & $h$ & $m$ & $n$ & $r$ & $w_{1}$ & $w_{2}$ \\
\hline (a) & 0.4 & 0.4 & 0.4 & 13.6 & 6.8 & 13.1 & 0.2 & 0.3 & 7.5 & 30 & 22 & Non & 1.9 & 0.85 \\
\hline (b) & Non & Non & Non & 13.6 & Non & 13.1 & 0.2 & 0.3 & 7.5 & 30 & 22 & Non & 1.9 & 0.85 \\
\hline (c) & 0.4 & 0.4 & 0.4 & 13.6 & 6.8 & 13.1 & Non & 0.3 & 7.5 & 30 & 22 & Non & 1.8 & 4 \\
\hline (d) & Non & Non & Non & 13.6 & Non & 13.1 & Non & 0.3 & 7.5 & 30 & 22 & Non & 1.8 & 4 \\
\hline (e) & 0.4 & 0.4 & 0.4 & Non & Non & Non & 0.2 & 0.3 & 7.5 & 30 & 25 & 7.8 & 2.2 & 3.7 \\
\hline (f) & Non & Non & Non & Non & Non & Non & 0.2 & 0.3 & 7.5 & 30 & 25 & 7.8 & 2.2 & 3.7 \\
\hline (g) & 0.4 & 0.4 & 0.4 & Non & Non & Non & Non & 0.3 & 7.5 & 30 & 25 & 7.8 & 1.8 & 10 \\
\hline (h) & Non & Non & Non & Non & Non & Non & Non & 0.3 & 7.5 & 30 & 25 & 7.8 & 1.8 & 10 \\
\hline
\end{tabular}

Unit: $\mathrm{mm}$

respectively. The SLR considered as the half part of the radiation patch is placed on the upper side of the whole patch. After initial numerical analysis, there is a huge impedance mismatch between the SLR and the conventional $50-\Omega \mathrm{CPW} /$ microstrip feed line. Therefore, the bottom part of the patch is used to match the impedance between the SLR and feed line. The whole radiation patch is printed on a $0.8 \mathrm{~mm}$ thick Rogers Ro4003 substrate (dielectric constant $\varepsilon_{r}=3.55$ and loss tangent $\left.\tan \delta=0.0027\right)$. For the antennas fed by CPW the ground plane and SLR are printed on the same side of the substrate, while for the antennas fed by microstrip line the ground plane is placed on the opposite side of the substrate. The dimensional parameters for the four kinds of antennas are finally optimized by finite element method based Ansoft HFSS software and concluded in Table 1. At the same time, the corresponding conventional singleband monopole antennas for the two kinds of feed lines are also designed with the same radiation patch size and ground plane size as shown in Figures 2(b), 2(d), 2(f), and 2(h). Finally, through standard printed circuit board fabrication techniques, all antennas are fabricated. In both simulation and measurement procedures, the $50-\Omega$ microminiature coaxial connectors (operated from DC to $6 \mathrm{GHz}$ ) are used to simulate and test the return loss and radiation characteristics for reducing the effects of connectors on the antennas.

\section{Results}

Firstly, the simulated and measured return loss properties of the four proposed SLR inspired antennas and four corresponding conventional antennas are shown in Figure 3. And the key characteristics for the four triple-band antennas are also concluded in Table 2. It can be known that the simulated and measured results shown in Figure 3 and concluded in Table 2 imply a good agreement. For the metamaterial inspired antennas, both the numerical and experimental results show three dips around $2.4,3.8$, and $4.9 \mathrm{GHz}$. The first operating frequency for these triple band antennas indicates a very narrow bandwidth compared to the following two operating frequencies, as shown in Figure 3 and Table 2. This is mainly due to the strong resonance characteristic of the SLR inspired radiation patches. For practical applications, we can expand the bandwidth by, for example, using low-dielectric-constant substrate with larger dielectric loss or adding wideband impedance matching circuits. In the higher frequency band $(4.5-6 \mathrm{GHz})$, there is slight frequency shift between numerical and experimental results for each kind of metamaterial inspired antenna. This shift mainly results from the mutual coupling introduced by connector and soldering in the experiment at high frequencies. To confirm this, we performed numerical simulations to show the effect of changing the connecter length on the return loss properties. One of the SLR inspired antennas with different connector length as shown in Figure 4(a) is chosen as an example. From Figures 4(b) and 4(c) it can be seen that, with increasing the connect length, the first two operating frequencies keep unchanged while the third one shifts to low frequency with worse return loss property. Therefore, for practical engineering applications, one should consider the connecter effect on the performance of such kind of antennas.

On the other hand, the simulated and measured reflection results for the conventional monopole antenna show only one transmission dip located in the range of $3.8-5 \mathrm{GHz}$. It means that our proposed metamaterial inspired antennas can operate at very lower frequency with the same antenna size for the conventional antenna. Therefore the proposed antennas have a highly compact size property. From the simulated and measured results, we can also know that, for both rectangular and circular shaped radiation patches and both CPW and microstrip feed methods, the tripleband operating properties can be flexibly achieved. It means that we can use any of the four antennas to integrate in the transmit/receive devices depending on the special space and arrangement requirements. Taking deep considerations in Table 2, the SLR inspired rectangular patch antennas have higher operating frequencies for $f_{1}$ and $f_{2}$ and lower frequency for $f_{3}$, compared with the circular patch antennas. This is because there is different resonance frequencies ratio for the rectangular and circular shaped SLR. So it provides a possible way to control each operating frequency by choosing different shaped resonators.

Figure 5 shows the simulated copolarization and crosspolarization radiation pattern characteristics for the four metamaterial inspired antennas at three corresponding reflection dips. It can be seen that, at the three frequencies, the four antennas exhibit typical eight-like radiation patterns in the copolarization E-plane, whereas in the copolarization $\mathrm{H}$ plane they possess nearly omnidirectional radiation patterns. Moreover, the cross-polarization radiations of the proposed antennas are very low for all the patterns. For examples, 


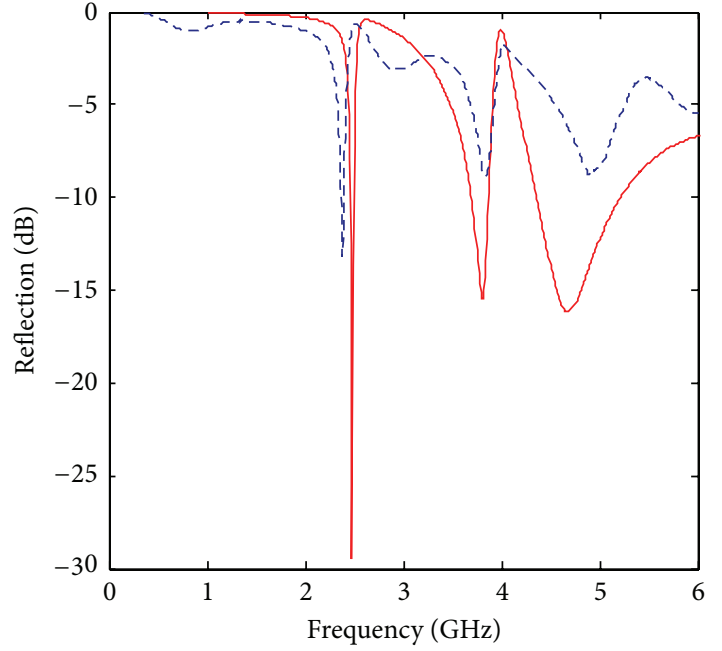

(a)

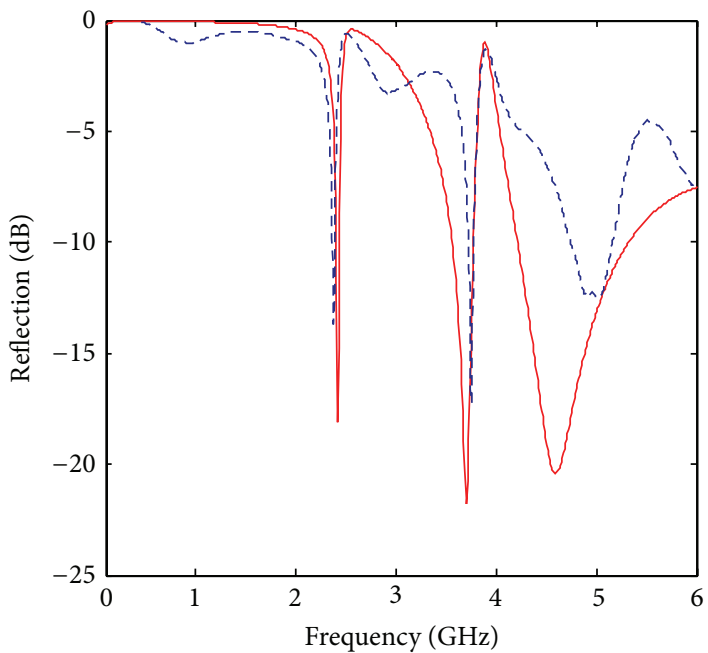

(c)

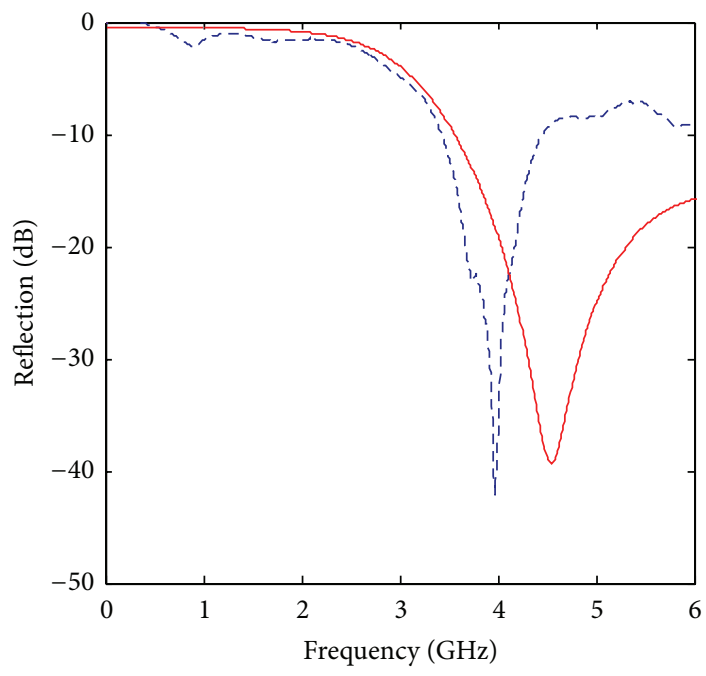

- Simulations

- - - Measurements

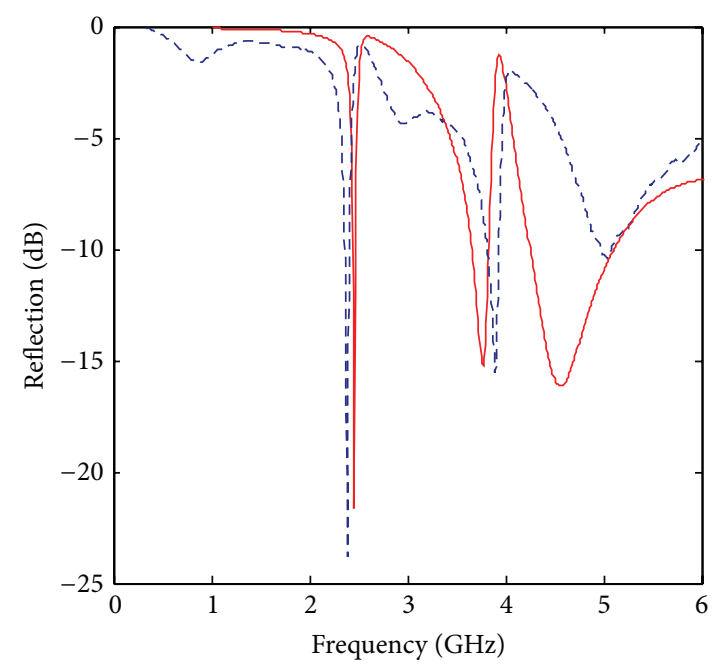

(b)

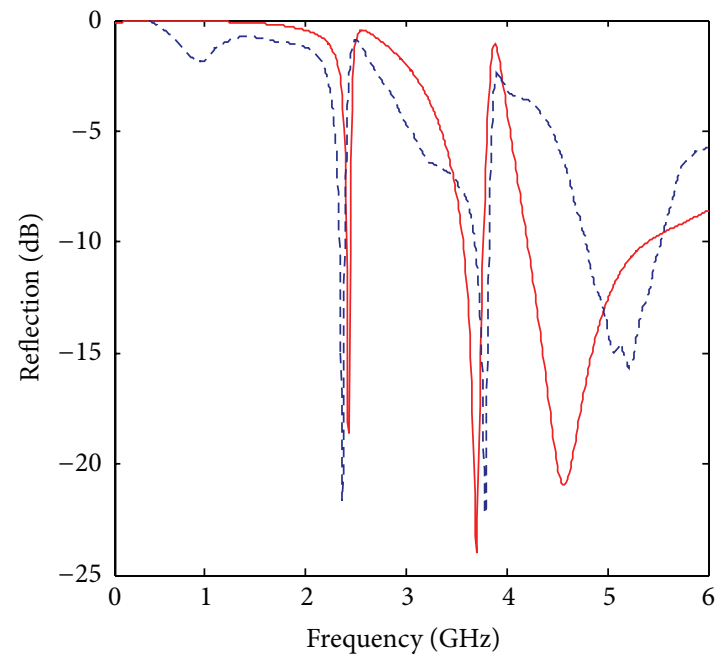

(d)

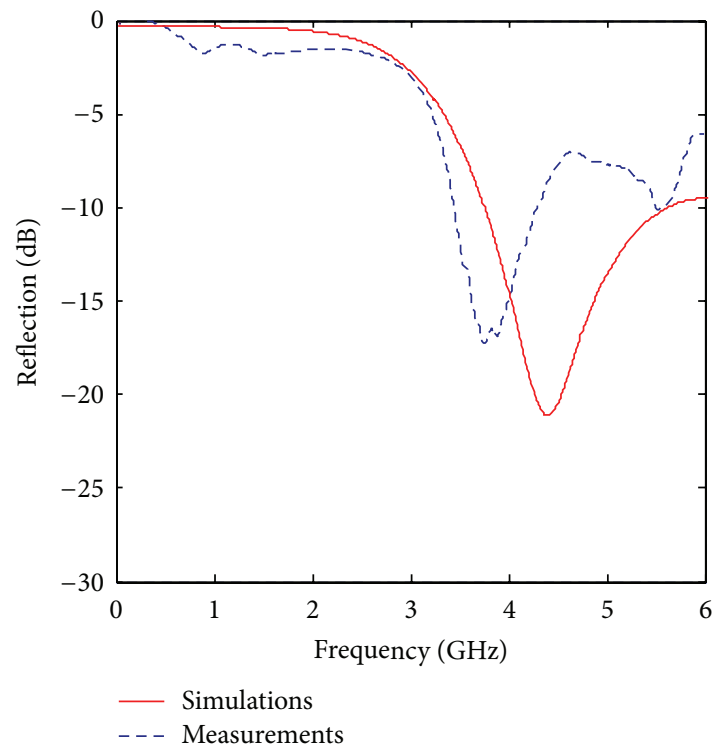

(f)

Figure 3: Continued. 


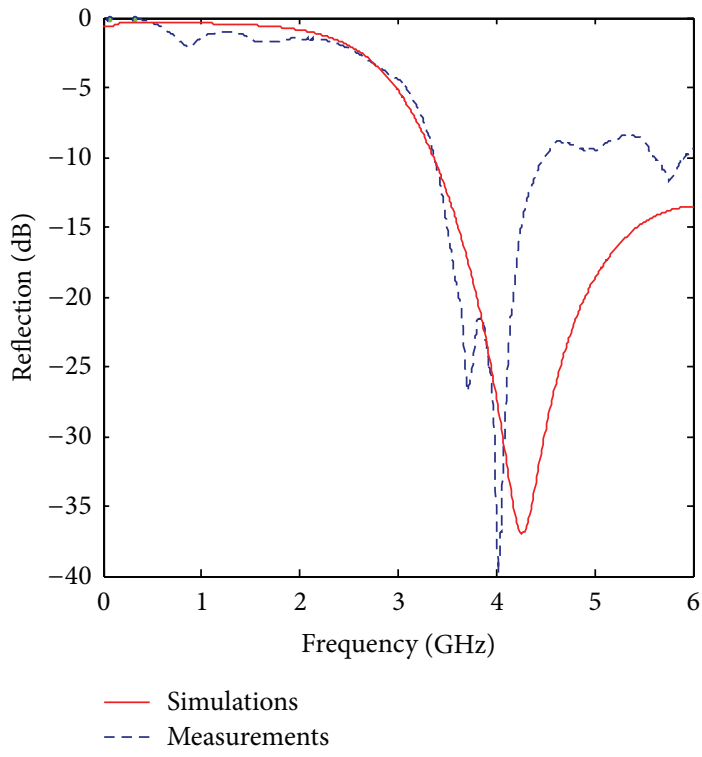

(g)

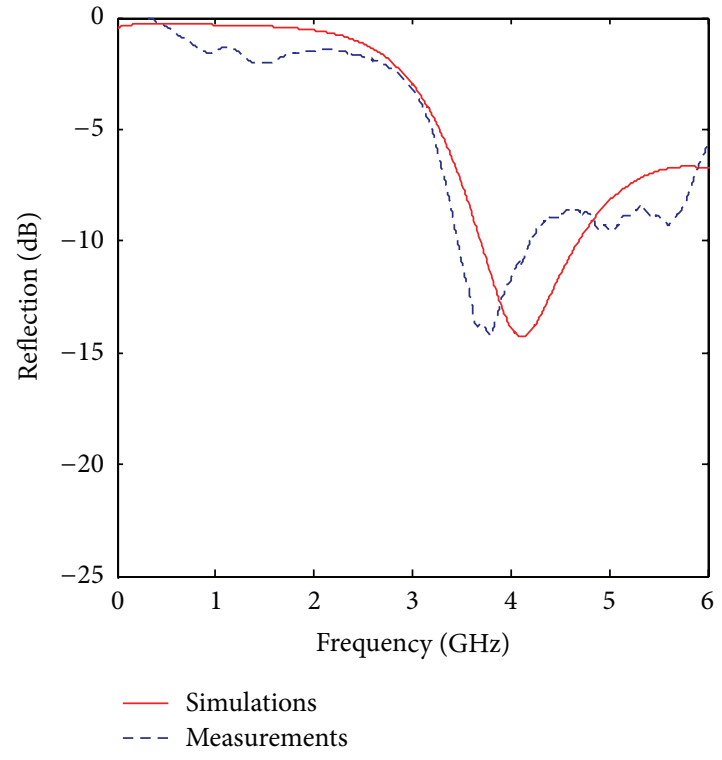

(h)

FIGURE 3: (a)-(d) Simulated and measured reflection properties of the four discussed SLR inspired triple-band antennas. (e)-(h) Simulated and measured reflection properties of the corresponding conventional single band antennas.

TABLE 2: Simulated and measured characteristics for the proposed SLR inspired triple-band antennas as shown in Figure 2.

\begin{tabular}{|c|c|c|c|c|c|c|c|}
\hline Antenna & & $f_{1}$ & $f_{2}$ & $f_{3}$ & Bandwidth for $f_{1}$ & Bandwidth for $f_{2}$ & Bandwidth for $f_{3}$ \\
\hline \multirow{2}{*}{ (a) } & Simulation & 2.48 & 3.8 & 4.67 & 0.003 & 0.1 & 0.47 \\
\hline & Measurement & 2.38 & 3.83 & 4.9 & 0.017 & 0.14 & 0.37 \\
\hline \multirow{2}{*}{ (c) } & Simulation & 2.46 & 3.76 & 4.55 & 0.01 & 0.1 & 0.45 \\
\hline & Measurement & 2.39 & 3.9 & 5.05 & 0.008 & 0.05 & 0.6 \\
\hline \multirow{2}{*}{ (e) } & Simulation & 2.42 & 3.7 & 4.6 & 0.015 & 0.05 & 0.32 \\
\hline & Measurement & 2.31 & 3.71 & 4.94 & 0.015 & 0.015 & 0.35 \\
\hline \multirow{2}{*}{ (g) } & Simulation & 2.43 & 3.7 & 4.57 & 0.017 & 0.04 & 0.29 \\
\hline & Measurement & 2.31 & 3.75 & 5.15 & 0.018 & 0.03 & 0.48 \\
\hline
\end{tabular}

Unit: GHz.

there is a $30 \mathrm{~dB}$ below the copolarization E-plane over $45^{\circ}$ range for the first antenna. And there is a $20 \mathrm{~dB}$ below the copolarization $\mathrm{H}$-plane over the omnidirectional angles, at all the three frequencies. These radiation patterns indicate an excellent polarization purity and such patterns are kept very well when integrated with metamaterial unit in the radiation patches. Moreover, it is seen that, for both the CPW and microstrip fed antennas, they have similar radiation patter at all the three frequencies. Therefore such kind of antenna can be integrated flexibly into other CPW and/or microstrip integrated circuits/devices.

To further characterize the designed antennas, we measured experimentally the radiation pattern, peak gain total, and efficiency in a commercial near field measurement system (Satimo SG 32) as shown in Figure 6, for the four SLR inspired antennas as comparisons. However, for the monopole antenna with such quite small ground plane, the measurement setup will affect mostly on the radiation pattern, due to the additional coaxial cable. As an example, Figure 7 shows the measured copolarization and crosspolarization radiation patterns for the SLR inspired circular patch triple-band antenna at corresponding frequencies. From Figure 7, it indicates that both E-plane and H-plane copolarization results show moderate distortions and worse cross-polarization characteristics, comparing to the numerical results shown in Figure 5. But we can still see the eight-like radiation patterns in the E-plane copolarization and omnidirectional radiation patterns in the $\mathrm{H}$-plane copolarization. As mentioned above, the difference between measured and simulated results is mainly from the connecting setup of the measurement system as shown in Figure 6. In our near future designs and measurements, such difference can be reduced by using more compact setup and adding some absorbers surrounding the feeding cables.

Table 3 concludes the measured peak gain total and efficiency at the three corresponding reflection dips for all the 


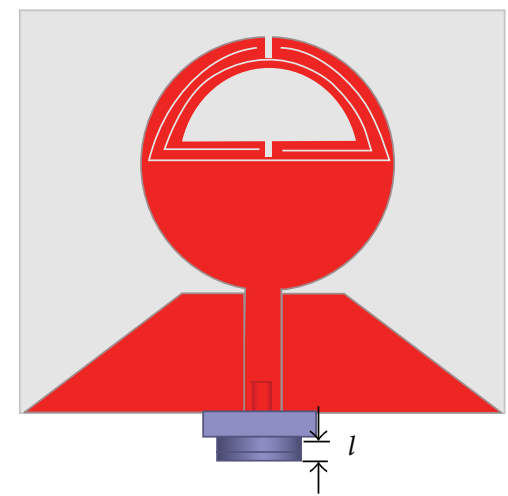

(a)

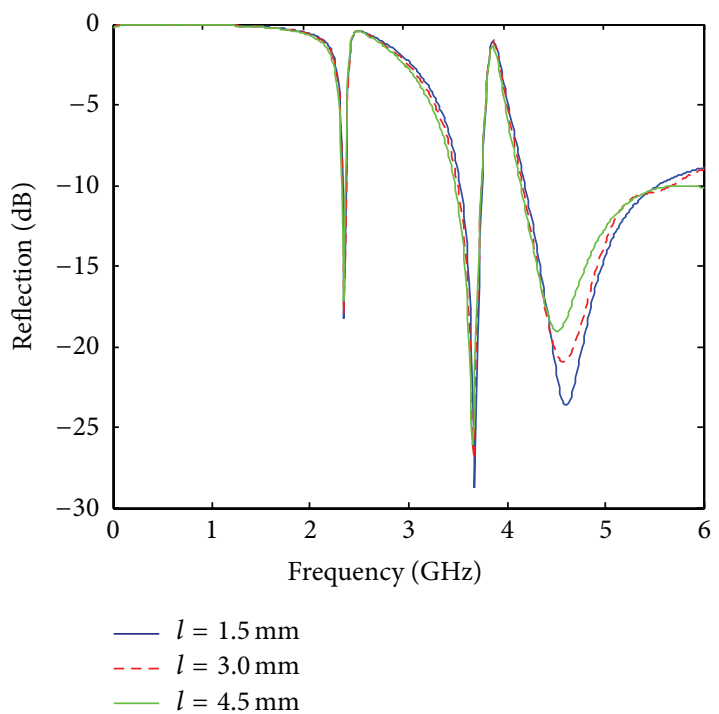

(b)

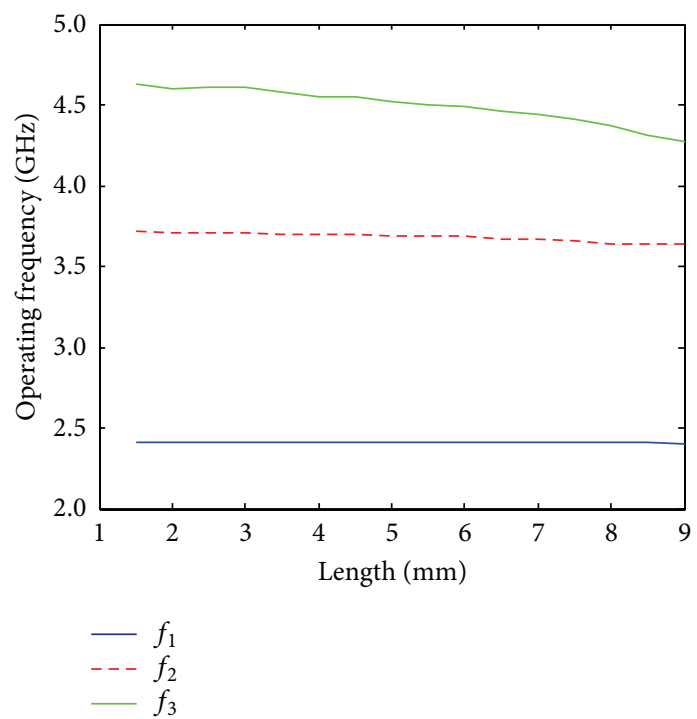

(c)

FIGURE 4: (a) One of the SLR inspired antennas with connector of length $l$. (b) Simulated reflection properties of the proposed antenna under different connecter length. (c) The three operating frequencies as a function of the connecter length.

TABLE 3: Measured peak gain total and efficiency properties for the proposed SLR inspired triple-band antennas as shown in Figure 2.

\begin{tabular}{lcccccc}
\hline Antenna & $\begin{array}{c}\text { Peak gain total } \\
\text { for } f_{1}\end{array}$ & $\begin{array}{c}\text { Peak gain total } \\
\text { for } f_{2}\end{array}$ & $\begin{array}{c}\text { Peak gain total } \\
\text { for } f_{3}\end{array}$ & Efficiency for $f_{1}$ & Efficiency for $f_{2}$ & Efficiency for $f_{3}$ \\
\hline (a) & 3.7 & 3.0 & 3.2 & 0.58 & 0.45 & 0.38 \\
(c) & 3.2 & 3.4 & 4.2 & 0.53 & 0.48 & 0.47 \\
(e) & 3.6 & 3.2 & 3.8 & 0.54 & 0.51 & 0.48 \\
(g) & 3.3 & 3.1 & 4.0 & 0.53 & 0.47 & 0.45 \\
\hline
\end{tabular}

Gain unit: dBic.

four SLR inspired antennas. It can be seen that all of the four antennas at each frequency have the same gain level. However, the measured gain total looks like higher than a typical omnidirectional monopole antenna. This is mainly because of the previously mentioned moderate distortions. Moreover, at higher operating frequency, the efficiency dropped down slightly. This is because the loss at such higher frequency is larger than the condition in the lower frequency. From the above simulated and measured results, however, we can still conclude such metamaterial inspired triple-band antennas can be easily used to modern wireless communications.
Comparing to the previously well-developed metamaterial inspired monopole antennas [12], our designs have near-same return loss and radiation performances while possessing simpler implementation method.

\section{Conclusions}

In this paper, comparing with the conventional monopole antennas, four kinds of novel metamaterial inspired miniaturized triple-band antennas consisting of a SLR as part of the radiation patch and fed with CPW and microstrip 


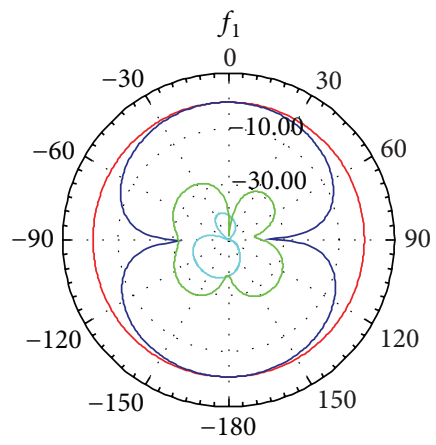

(a)

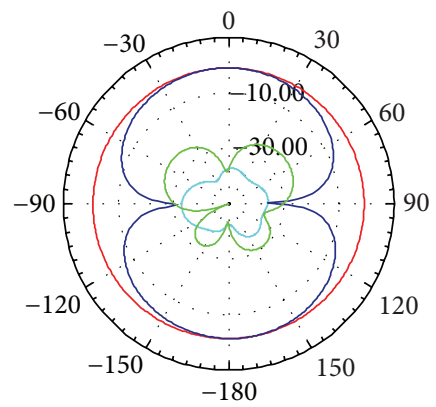

(d)

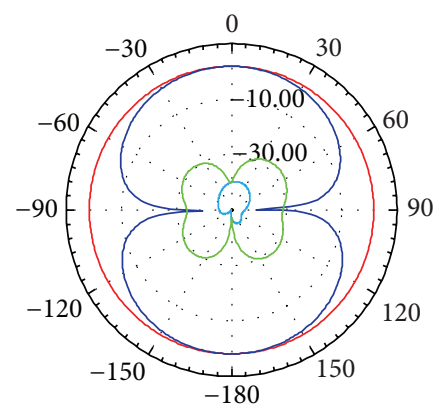

(g)

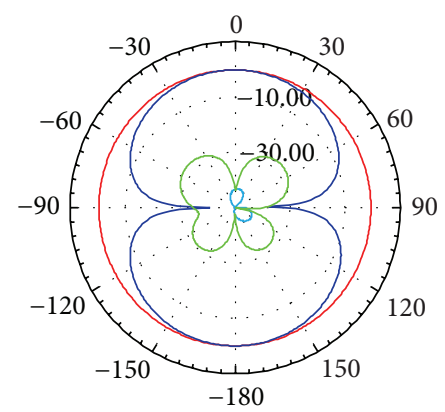

E-plane copolarization
E-plane cross-polarization
H-plane copolarization
H-plane cross-polarization

(j)

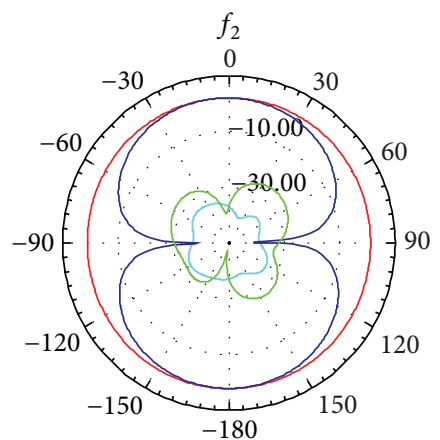

(b)

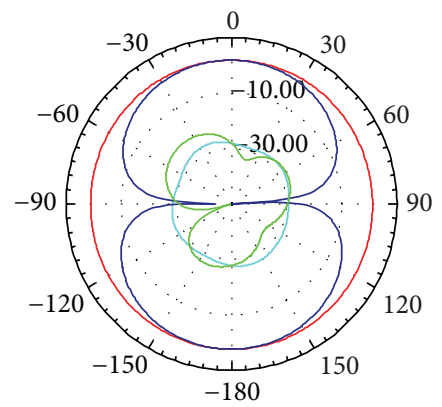

(e)

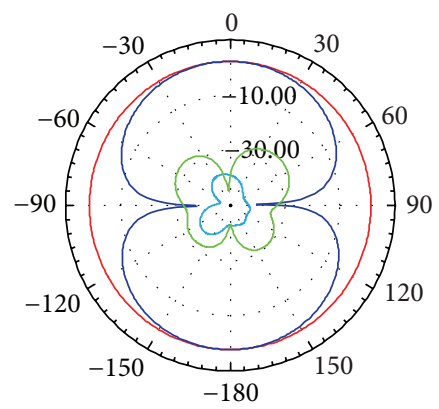

(h)
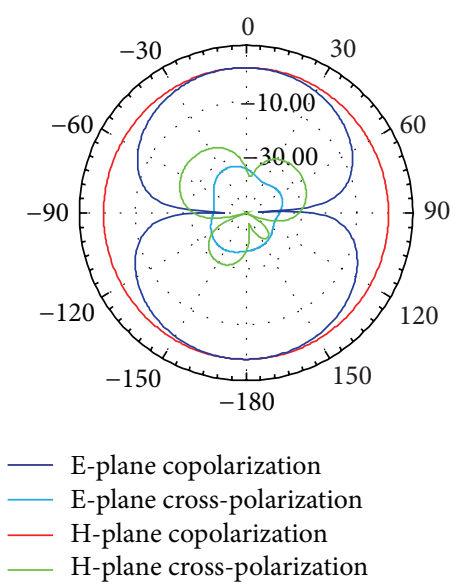

(k)

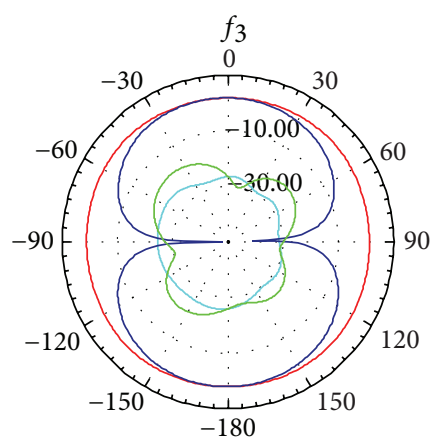

(c)

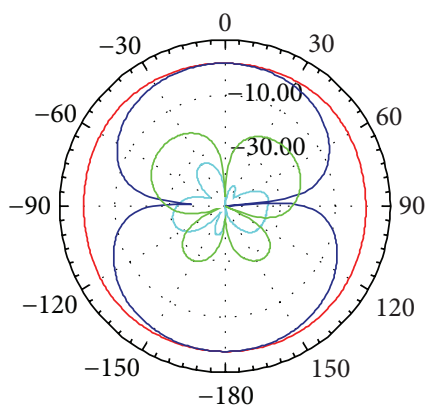

(f)

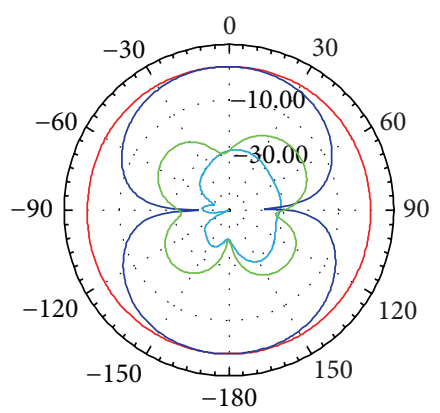

(i)
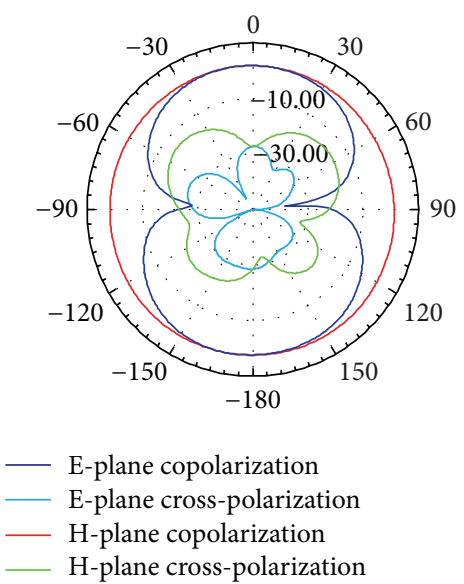

(l)

FIGURE 5: Simulated E-plane and H-plane copolarization and cross-polarization radiation patterns for the proposed four SLR inspired tripleband antennas. (a)-(c) CWP fed squared patch antennas, (d)-(f) microstrip fed squared patch antennas, (g)-(i) CWP fed circular patch antennas, and (j)-(l) microstrip fed circular patch antennas. 


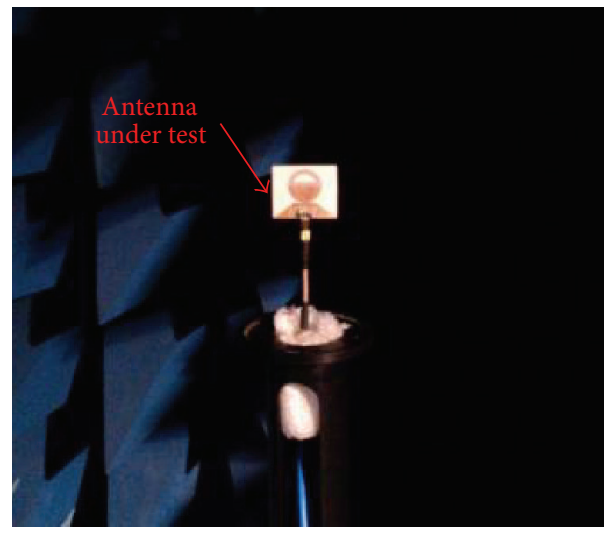

Figure 6: Photograph of the Satimo SG 32 near field measurement setup and the triple-band antenna under test.

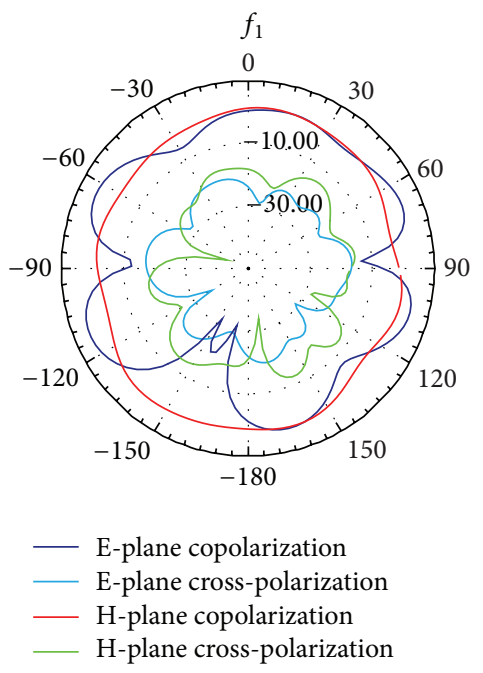

(a)
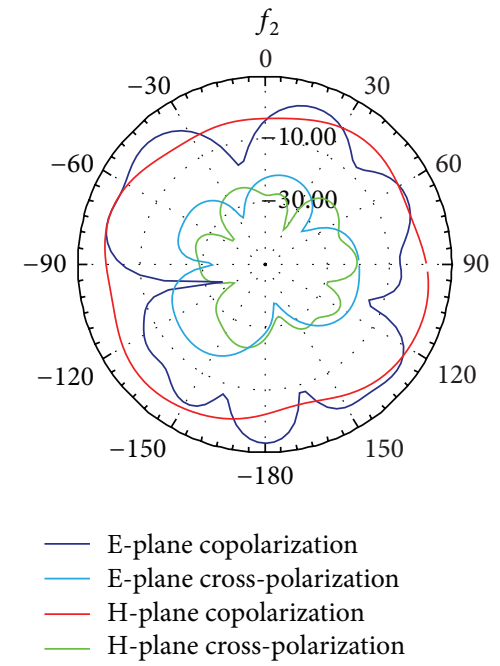

(b)

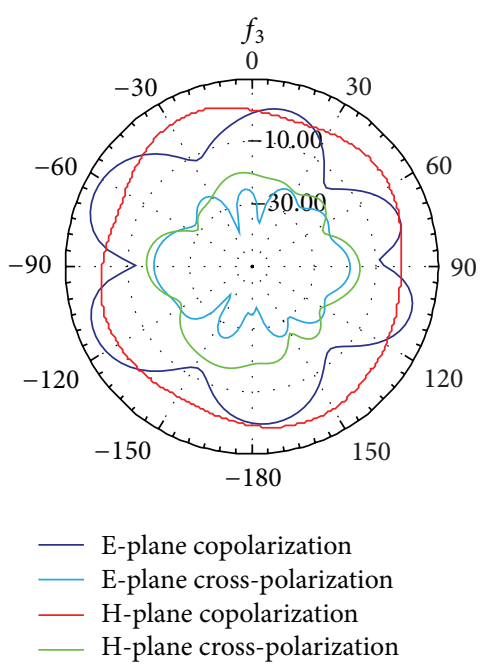

(c)

Figure 7: Measured E-plane and H-plane copolarization and cross-polarization radiation patterns for the selected CWP fed circular SLR inspired triple-band antennas at three operating frequencies.

transmission lines, respectively, are discussed through numerical simulations and experimental demonstrations. The proposed antennas exhibit comparable radiation pattern with the conventional monopole antennas, including very well eight-like radiation patterns and low cross-polarizations and high peak gain total and efficiency. Such antennas have potential applications for modern wireless communications and can be integrated flexibly into other CPW and/or microstrip integrated circuits.

\section{Conflict of Interests}

The authors declare that there is no conflict of interests regarding the publication of this paper.

\section{Acknowledgments}

This work was supported in part by the National Natural Science Foundation of China (Grant no. 61371047) and by Research Fund for the Doctoral Program of Higher Education of China (Grant no. 20110185110014). Yongjun Huang also gratefully acknowledges the Scholarship Award for Excellent Doctoral Student granted by Ministry of Education of China (Grant no. A03003023901006).

\section{References}

[1] M. Niroo-Jazi and T. A. Denidni, "A new triple-band circular ring patch antenna with monopole-like radiation pattern using a hybrid technique," IEEE Transactions on Antennas and Propagation, vol. 59, no. 10, pp. 3512-3517, 2011.

[2] J.-H. Yoon, Y.-C. Rhee, and W.-S. Kim, "A rectangular ring, open-ended monopole antenna with two symmetric strips for WLAN and WiMAX applications," International Journal of Antennas and Propagation, vol. 2013, Article ID 109450, 9 pages, 2013.

[3] Y.-T. Wan, D. Yu, and F.-S. Zhang, "Miniature multi-band monopole antenna using spiral ring resonators for radiation pattern characteristics improvement," Electronics Letters, vol. 49, no. 6, pp. 382-384, 2013.

[4] P. Wang, G.-J. Wen, Y.-J. Huang, and Y.-H. Sun, "Compact CPW-fed planar monopole antenna with distinct triple bands 
for WiFi/WiMAX applications," Electronics Letters, vol. 48, no. 7, pp. 357-359, 2012.

[5] J. Wang and X. He, "Analysis and design of a novel compact multiband printed monopole antenna," International Journal of Antennas and Propagation, vol. 2013, Article ID 694819, 8 pages, 2013.

[6] J. T. Rayno and S. K. Sharma, "Compact spirograph planar monopole antenna covering $\mathrm{C}$-/X-band with invariant radiation pattern characteristics," IEEE Transactions on Antennas and Propagation, vol. 60, no. 12, pp. 6002-6007, 2012.

[7] D. R. Smith, W. J. Padilla, D. C. Vier, S. C. Nemat-Nasser, and S. Schultz, "Composite medium with simultaneously negative permeability and permittivity," Physical Review Letters, vol. 84, no. 18, pp. 4184-4187, 2000.

[8] R. W. Ziolkowski, P. Jin, and C.-C. Lin, "Metamaterial-inspired engineering of antennas," Proceedings of the IEEE, vol. 99, no. 10, pp. 1720-1731, 2011.

[9] D. Jiang, Y. Xu, R. Xu, and W. Lin, "Compact dual-bandnotched UWB planar monopole antenna with modified CSRR," Electronics Letters, vol. 48, no. 20, pp. 1250-1252, 2012.

[10] L.-M. Si, W. Zhu, and H.-J. Sun, "A compact, planar, and CPWfed metamaterial-inspired dual-band antenna," IEEE Antennas and Wireless Propagation Letters, vol. 12, pp. 305-308, 2013.

[11] L.-M. Si and X. Lv, "CPW-FED multi-band omni-directional planar microstrip antenna using composite metamaterial resonators for wireless communications," Progress in Electromagnetics Research, vol. 83, pp. 133-146, 2008.

[12] J. Zhu, M. A. Antoniades, and G. V. Eleftheriades, "A compact tri-band monopole antenna with single-cell metamaterial loading," IEEE Transactions on Antennas and Propagation, vol. 58, no. 4, pp. 1031-1038, 2010.

[13] E. Ekmekci and G. Turhan-Sayan, "Single loop resonator: dualband magnetic metamaterial structure," Electronics Letters, vol. 46, no. 5, pp. 324-325, 2010.

[14] O. Yurduseven, A. E. Yilmaz, and G. Turhan-Sayan, “Triangular-shaped single-loop resonator: a triple-band metamaterial with MNG and ENG regions in S/C bands," IEEE Antennas and Wireless Propagation Letters, vol. 10, pp. 701-704, 2011.

[15] O. Yurduseven, A. E. Yilmaz, and G. Turhan-Sayan, "Hybridshaped single-loop resonator: a four-band metamaterial structure," Electronics Letters, vol. 47, no. 25, pp. 1381-1382, 2011.

[16] R. Wen, "Compact planar triple-band monopole antennas based on a single-loop resonator," Electronics Letters, vol. 49, no. 15, pp. 916-918, 2013.

[17] J. Zhong, F. Wang, G. Wen et al., “Tunable triple-band negative permeability metamaterial consisting of single-loop resonators and ferrite," Journal of Electromagnetic Waves and Applications, vol. 27, no. 3, pp. 267-275, 2013. 

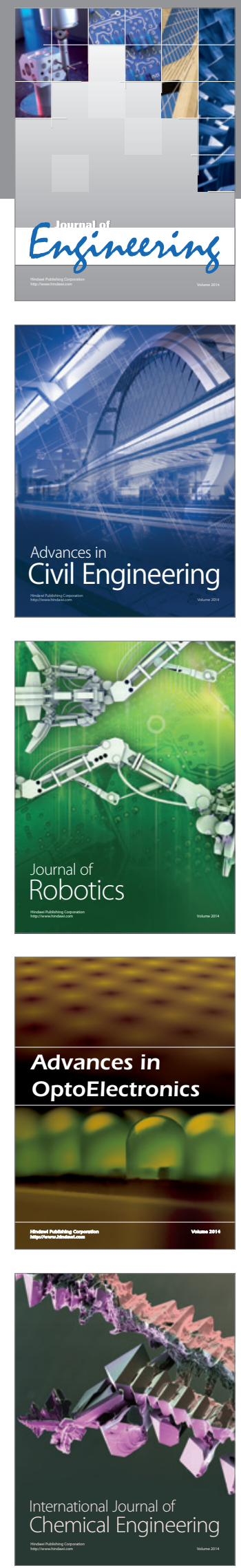

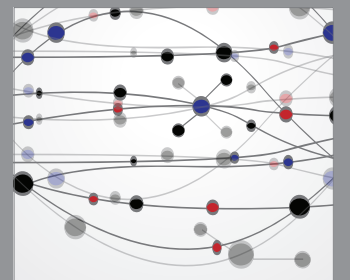

The Scientific World Journal
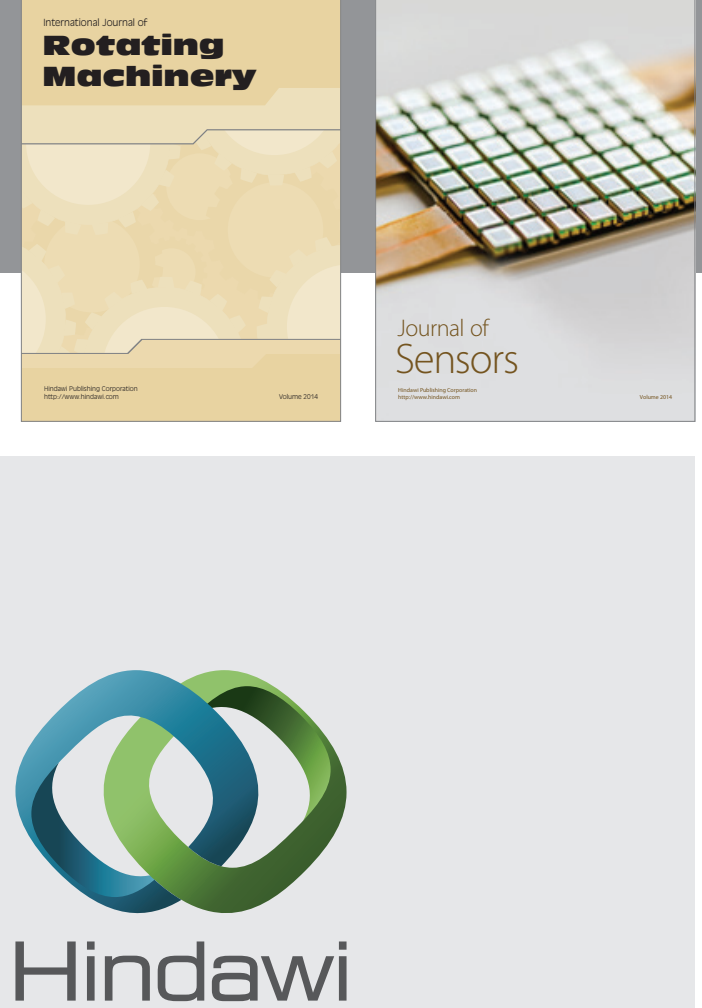

Submit your manuscripts at http://www.hindawi.com
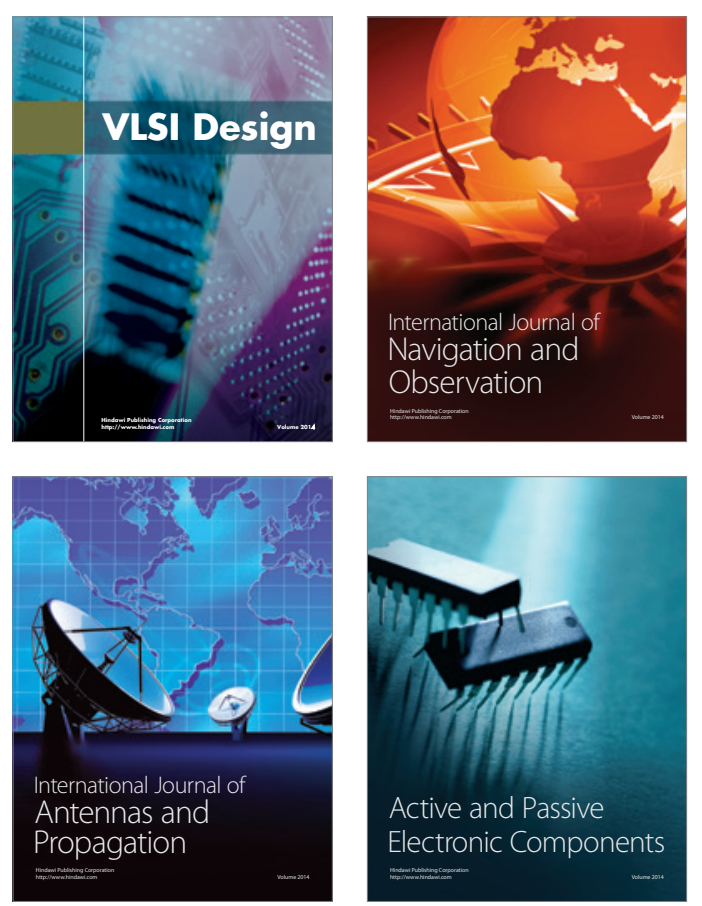
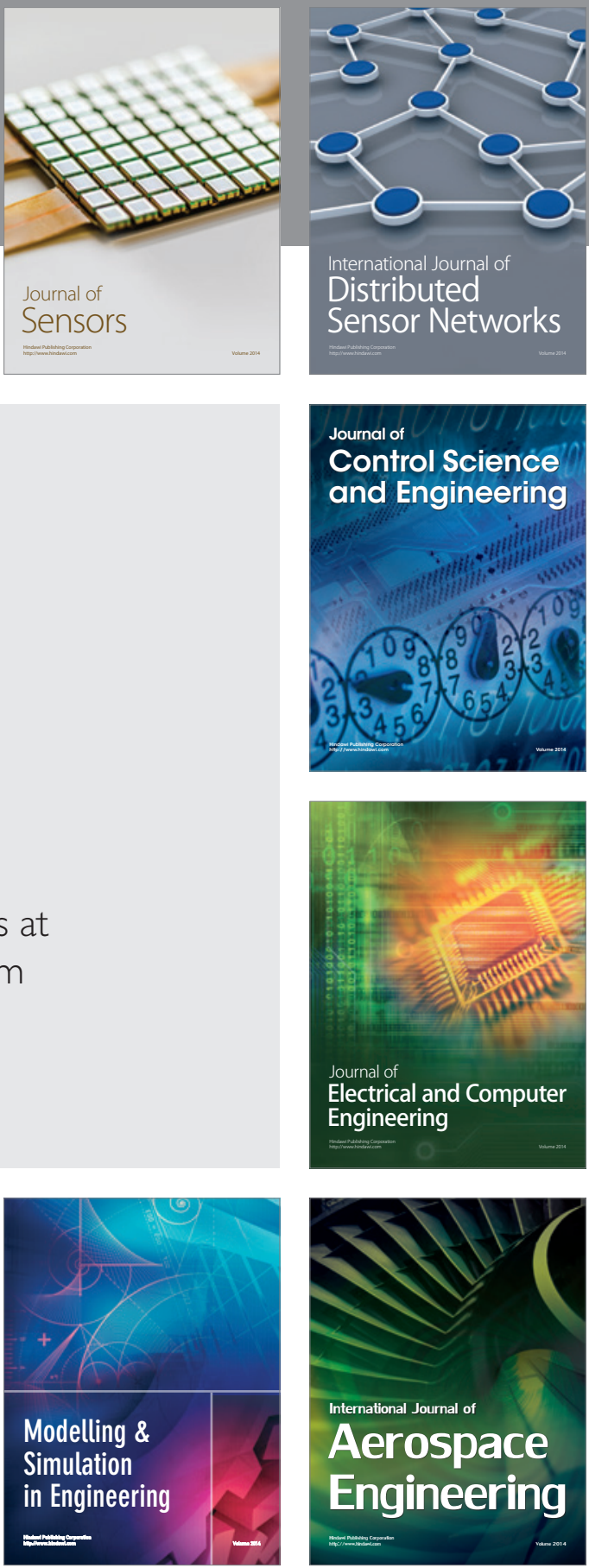

Journal of

Control Science

and Engineering
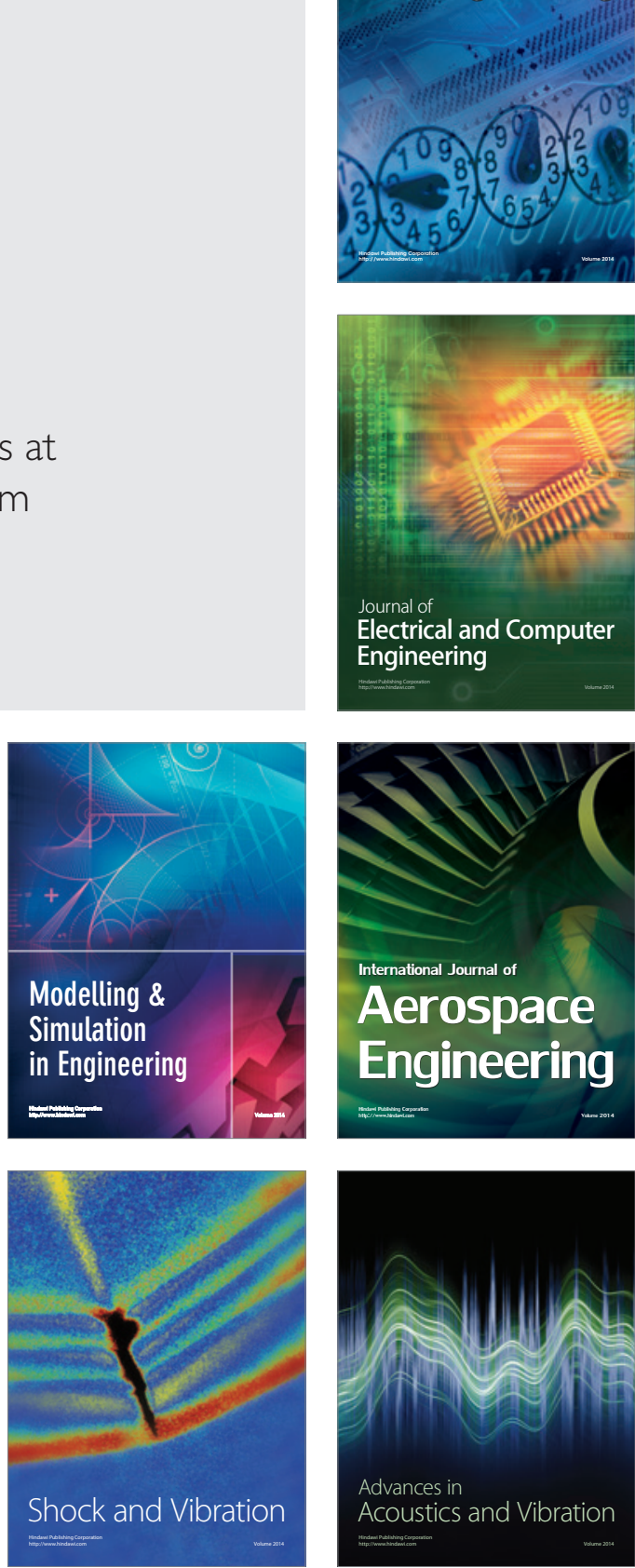